\title{
Spontaneous VEGF Production by Cultured Peritoneal Mesothelial Cells from Patients on Peritoneal Dialysis
}

Long-term peritoneal dialysis (PD) may result in

increased peritoneal vascularization secondary to

neovascularization (1). Vascular endothelial growth

factor (VEGF) is the main factor known to participate

in new vessel formation (2). Peritoneal VEGF

production has to be an etiologic factor in peritoneal

neovascularization due to the relatively higher levels

found in peritoneal effluent than in plasma (3).

The exact origin of peritoneal effluent VEGF is not

known. Recently, pleural mesothelial cells (MC) have

been shown to produce small amounts of VEGF (4).

Also, VEGF has been detected in peritoneal capillary

endothelium and surrounding tissue in long-term PD

patients (5). Finally, the glucose degradation product

(GDP) carboxymethyl lysine has been demonstrated

to be co-localized with VEGF in the mesothelial layer

in chronic PD patients (6). These authors have also

shown the production of $\mathrm{VEGF}$ by rat $\mathrm{MC}$ exposed

to GDP.

Many usual conditions intrinsic to PD are related

to the VEGF present in the peritoneum in these patients.

Our hypothesis is that human MC might be a

source for VEGF. To study their potential VEGF production,

we determined VEGF levels in conditioned

medium during ex vivo culture of MC isolated from

the peritoneal effluent of active PD patients.

PATIENTS AND METHODS

Patients and Peritoneal Characteristics: We assessed

peritoneal antecedents and peritoneal function

by determining the mean number of prior

peritonitis episodes and the peritoneal transport parameters

[mass transfer coefficients (MTC) of urea

and creatinine and net ultrafiltration during a 4-hour

kinetic study with $2.27 \%$ glucose] (7) in 21 patients.

In addition, the accumulated peritoneal dose of glucose

administered during the complete PD period was

calculated for each patient.

Mesothelial cells (8) were isolated from nocturnal

peritoneal effluent incubated in hanging PD bags for

3 - 4 hours at $37^{\circ} \mathrm{C}$. The residual effluent was removed

by vacuum suction with a sterile pipette, leaving approximately

$200 \mathrm{~mL}$ in the bottom. Cells were resuspended

in this volume, transferred to four 50-mL

tubes, and washed twice with phosphate-buffered

saline by centrifugation at 1500 rpm for 20 minutes.

Then they were seeded in 25-cm2 tissue culture flasks

and incubated at $37^{\circ} \mathrm{C}$ in a humidified atmosphere

with 5\% $\mathrm{CO}_{2}$, and maintained in M-199 (Biological

Industries, Kibbutz Beit Haemek, Israel) supplemented with $20 \%$ fetal bovine serum, 100

$\mathrm{IU} / \mathrm{mL}$ penicillin,

$100 \mu \mathrm{g} / \mathrm{mL}$ streptomycin, and 2\% Biogro-2 (containing

insulin, transferrin, ethanol amine, and

putrescine) (Biological Industries). After reaching

confluence, cells were detached with trypsin/EDTA, 
resuspended, and seeded in 24-well plates at $2 \times 104$ cells/well. Incubation was performed as before; the medium was renewed every 3 - 4 days.

None of the cultures used to evaluate VEGF production showed bacterial or fungal contamination.

Mesothelial cells were identified at isolation from effluent

and after trypsinization. Morphology was assessed

in cytospin optical microscopy slides (500 rpm)

stained with May-Grünwald-Giemsa. Mesothelial

cells were thus easily distinguished from macrophages,

lymphocytes, and neutrophils by their morphological

features defined under light microscope. No

atypical or giant cells were observed in these cultures.

Mesothelial cells were characterized by immunohistochemical

staining, identifying $M C$ by their positive

staining for cytokeratin and negative staining for

CD45 (hematopoietic cells), HLA-DR (macrophages

and lymphocytes), and $\operatorname{VWF}$ (endothelial cells). All

antibodies were from Dako (Glostrup, Denmark).

VEGF165 Determinations: VEGF was measured in

the supernatant of 21 cultures of MC. VEGF determination

in supernatant was performed at day 13 of

seeding in 24-well plates, since the proliferation plateau

usually starts at this time while MC simultaneously

form a monolayer reaching confluence. This

time avoids vigorous cell proliferation, which is a consequence

of cell subconfluence state. After 48 hours

of serum deprivation, supernatant was taken for

analysis. VEGF165 was determined by enzyme immunoassay

(ELISA) with a kit from R\&D Systems, Inc.

(Minneapolis, MN, U.S.A.).

Statistical Analysis: Data are expressed as mean \pm

SD. The Mann-Whitney test was used for data comparisons

as a nonnormal distribution of the results

was found. Regression analysis was performed with

the spearman test.

RESULTS

VEGF was detected in the supernatant from all

21 independent cultures of MC taken from PD bags

and grown in culture. All the values observed were

within the detection range for the ELISA kit, with

mean VEGF levels of $548 \pm 518 \mathrm{pg} / \mathrm{mL}$ (range 59 -

$1747 \mathrm{pg} / \mathrm{mL})$. When peritoneal data and cell counts

from quartile 25 and quartile 75 were compared, no

significant differences were found (Table 1). Although

VEGF is also a vascular permeability factor, the levels

found in conditioned medium from MC in culture

are not related to peritoneal permeability to small

molecules.

Due to the wide range of values, the entire group

was artificially divided for supernatant VEGF levels

higher $(\mathrm{n}=12)$ and lower $(\mathrm{n}=9)$ than $200 \mathrm{pg} / \mathrm{mL}$.

There was a trend toward a longer stay on PD in patients

with lower supernatant levels. The mesothelial

cell count on day 13 was similar for the two groups

$(65699 \pm 28738$ vs $74930 \pm 39245, \mathrm{p}=0.88)$; the

accumulated peritoneal glucose load was also similar

$(72.06 \pm 70.73 \mathrm{~kg}$ vs $122.41 \pm 211.78 \mathrm{~kg}, \mathrm{p}=0.86)$. 
We found no overall correlation between supernatant VEGF levels and age, sex, time on PD, solute transport characteristics (Table 2), ultrafiltration rate, or accumulated dose of glucose. The MC count at day 13 was not significantly correlated with VEGF levels in the supernatant $(r=-0.33, p=0.13, \mathrm{NS})$. Furthermore, correction of supernatant VEGF levels according to the cell density at day 13 showed no significant correlation with any of the independent variables. We found no differences in supernatant VEGF levels between the different types of MC morphologies. DISCUSSION

In the present study, we report the capacity of peritoneal MC, cultured in vitro, to produce VEGF, suggesting that MC may contribute to the neoangiogenesis observed in some PD patients. This study represents a direct demonstration of VEGF production by human peritoneal MC in culture, and provides an explanation for the significant amounts of VEGF found in peritoneal effluent (3) and for its presence at the mesothelial level (6). However, these findings do not demonstrate the constitutive production of VEGF by MC from nondialyzed peritoneum. VEGF production by MC might be the result of an interaction with the continuously administered dialysate. In fact, pleural MC in culture from patients with cardiac insufficiency produce VEGF in amounts below the range of technique detection (4), much lower than the levels we have found in peritoneal MC. The extreme differences between the VEGF levels produced by each cell population could be determined by the particular situation of peritoneal MC in PD patients. The amounts produced by peritoneal MC are closer to the amounts produced by malignant pleural MC than to those produced by nonmalignant cells (4). No information has been found on VEGF production by MC lines.

Although other peritoneal cells, such as macrophages, are able to produce VEGF (9), there is no possibility of interference by these cells in our study. By our methodology (8), macrophages initially collected from the peritoneal effluent are progressively eliminated by washing, as they do not adhere to the flask surface. 800

We have demonstrated by analysis of supernatant from cultured MC that peritoneal MC can produce VEGF. This production is entirely spontaneous, although we do not know whether it might be a response to other stimuli. VEGF supernatant levels were independent of the growth rate and number of MC. There was a tendency toward an inverse relationship between this number and the VEGF level, but it was not statistically significant. The great variation in levels found in our series is remarkable and is not explained by any of the variables examined. The analysis by extreme groups of values showed no significant differences. The morphology of MC observed 
after their growth in T-25 flasks did not differentiate

cells according to their VEGF-production capacity.

In consideration of the large amounts of glucose

present in the peritoneal cavity of PD patients, we

analyzed the impact of cumulative glucose overload

on VEGF levels during the whole PD period. However,

glucose overload was not correlated with supernatant

VEGF levels. It is necessary to involve other

complementary factors in this potentially pathogenic

pathway. High glucose levels are the main factor implicated

in ocular angiogenesis in diabetic patients,

mediated by VEGF overexpression by retinal epithelial

cells (10).

In conclusion, our study demonstrates the ex vivo

VEGF production by peritoneal mesothelial cells from

PD patients. The great difference with respect to pleural

mesothelial cells in the capacity of VEGF synthesis

suggests that, possibly, factors related to PD

influence this production. It is necessary to carry the

research further and explain why mesothelial cells

produce different amounts of VEGF.

Rafael selgas*

Gloria del Pesol

M.-Auxiliadora Bajol

M.-Angeles Castrol

Susana Molina2

Antonio Cirugeda

José A. Sánchez-Tomero

M.-José Castrol

Vicente Alvarez

Angel Corbíz

Francisco Varaz

Nephrology Service

Hospital Universitario La Princesa

TABLE 2

Individual Values of Supernatant VEGF and

Peritoneal Mass Transfer Coefficients (MTC)

of Urea and Creatinine

Urea MTC Creatinine MTC VEGF level VEGF corrected

$(\mathrm{mL} / \mathrm{min})(\mathrm{mL} / \mathrm{min})(\mathrm{pg} / \mathrm{mL})$ by \# cells

$25.7 \quad 11.0 \quad 1448 \quad 70.2$

$17.5 \quad 9.9 \quad 175 \quad 9.7$

$19.3 \quad 13.0 \quad 662 \quad 9.3$

$23.5 \quad 9.2 \quad 558 \quad 7.4$

$\begin{array}{lllll}27.2 & 10.8 & 780 & 11.6\end{array}$

$\begin{array}{lllll}43.3 & 26.2 & 125 & 1.7\end{array}$

$21.6 \quad 8.5 \quad 184 \quad 2.9$

$\begin{array}{lllll}22.9 & 14.2 & 483 & 5.1\end{array}$

$35.7 \quad 14.2 \quad 1369 \quad 11.7$

$20.6 \quad 6.0 \quad 837 \quad 13.0$

$\begin{array}{llll}18.0 & 9.5 & 193 & 3.5\end{array}$

$\begin{array}{lllll}25.9 & 16.4 & 285 & 3.1\end{array}$

$14.8 \quad 8.6 \quad 639 \quad 8.5$

$24.0 \quad 12.7 \quad 59 \quad 1.2$

$\begin{array}{lllll}12.8 & 7.0 & 59 & 0.4\end{array}$

$16.5 \quad 10.2 \quad 1747 \quad 69.9$

$\begin{array}{llll}17.5 & 6.9 & 172 & 1.9\end{array}$

$10.6 \quad 2.1 \quad 127 \quad 1.3$

$\begin{array}{lllll}24.0 & 9.2 & 1340 & 29.4\end{array}$

$20.0 \quad 10.6 \quad 93 \quad 1.4$

$17.4 \quad 6.2686 \quad 16.9$

VEGR = vascular endothelial growth factor

Normal ranges: urea MTC 18 - $22 \mathrm{~mL} / \mathrm{minute;}$ creatinine

MTC 7 - $11 \mathrm{~mL} / \mathrm{minute.}$ 
TABLE 1

Characteristics of Patients with Extreme VEGF Supernatant Levels

VEGF quartile 25 VEGF quartile 75 p Value

Supernatant VEGF (pg/mL) $92.7 \pm 33.7 \quad 1348 \pm 328<0.01$

Mesothelial cells (N) $89250 \pm 42 \quad 663 \quad 54 \quad 513 \pm 39 \quad 006 \quad 0.21$

Age (years) $61.1 \pm 8.6 \quad 55 \pm 21 \quad 0.57$

Months on PD $54.1 \pm 88 \quad 21.5 \pm 19 \quad 0.4$

Episodes of peritonitis $1.4 \pm 2.6 \quad 0.8 \pm 1.3 \quad 0.6$

Accumulated glucose load (kg) $156.1 \pm 287 \quad 84.3 \pm 66 \quad 0.6$

Creatinine MTC (mL/min) $11.7 \pm 9 \quad 10.1 \pm 3 \quad 0.7$

Urea MTC (mL/min) $22.1 \pm 13 \quad 24.4 \pm 7.1 \quad 0.7$

Ultrafiltration (mL/4 hr, 2.27\% glucose) $650 \pm 339540 \pm 1240.5$

VEGR = vascular endothelial growth factor; $\mathrm{PD}=$ peritoneal dialysis; $\mathrm{MTC}=$ mass transfer coefficient.

\section{Nephrology Service 1}

Hospital Universitario La Paz

Department of Biochemistry2

Faculty of Medicine

Universidad Autónoma

Consejo superior de Investigaciones Científicas

for the "Group of Peritoneal Studies in Madrid"

Instituto Reina Sofía de Investigaciones

Nefrológicas de la Fundación Renal Iñigo

Alvarez de Toledo

Madrid, spain

*email: rselgasehlpr.insalud.es

REFERENCES

1. Mateijsen MAM, Van der Wal A, Hendriks PMEM,

Zweers MM, Mulder J, Striujk DG, et al. Vascular and

interstitial changes in the peritoneum of CAPD patients

with peritoneal sclerosis. Perit Dial Int 1999;

$19: 517-25$.

2. Ferrara N, Davis-Smyth T. The biology of vascular endothelial growth factor. Endocr Rev 1997; 18:3-22. 3. Zweers MM, Waart DR, Smit W, Struijk DG, Krediet RT. Growth factors VEGF and TGF-betal in peritoneal dialysis. J Lab Clin Med 1999; 134:124-32.

4. Kumar-Singh S, Weyler J, Martin MJ, Vermeulen PB, Van Marck E. Angiogenic cytokines in mesothelioma:

a study of VEGF, FGF-1 and -2 , and TGF $\beta$ expression.

J Pathol 1999; 189:72-8.

5. Combet S, Miyata T, Moulin P, Pouthier D, Goffin E, Devuyst $O$. Vascular proliferation and enhanced expression of endothelial nitric oxide synthase in human peritoneum exposed to long-term peritoneal dialysis. J Am Soc Nephrol 2000; 11:717-28.

6. Inagi R, Miyata T, Yamamoto T, Suzuki D, Urakami K, Saito A, et al. Glucose degradation product methylglyoxal enhances the production of vascular endothelial growth factor in peritoneal cells: role of the functional and morphological alteration of peritoneal membranes in peritoneal dialysis. FEBS Lett 1999; $463: 260-4$.

7. Selgas R, Fernández-Reyes MJ, Bosque E, Bajo MA, Borrego F, Jiménez C, et al. Functional longevity of the human peritoneum: how long is continuous peritoneal dialysis possible? Results of a prospective medium longterm study. Am J Kidney Dis 1994; 23:64-73.

8. Díaz C, Selgas R, Castro MA, Bajo MA, Fernández de

Castro M, Molina S, et al. Ex vivo proliferation of mesothelial

cells directly obtained from peritoneal effluent:

its relationship with peritoneal antecedents and functional

parameters. Adv Perit Dial 1998; 14:19-24.

9. McLaren J, Prentice A, Charnock-Jones DS, Millican

$\mathrm{SA}$, Muller $\mathrm{KH}$, Sharkey AM, et al. Vascular endothelial growth factor is produced by peritoneal fluid macrophages in endometriosis and is regulated by ovarian steroids. J Clin Invest 1996; 98:482-9. 
10. Aiello LP, Avery RL, Arrigg PG, Keyt BA, Jampel HD, Shah ST, et al. Vascular endothelial growth factor in ocular fluid of patients with diabetic retinopathy and other retinal disorders. N Engl J Med 1994; 331:1480-7. 